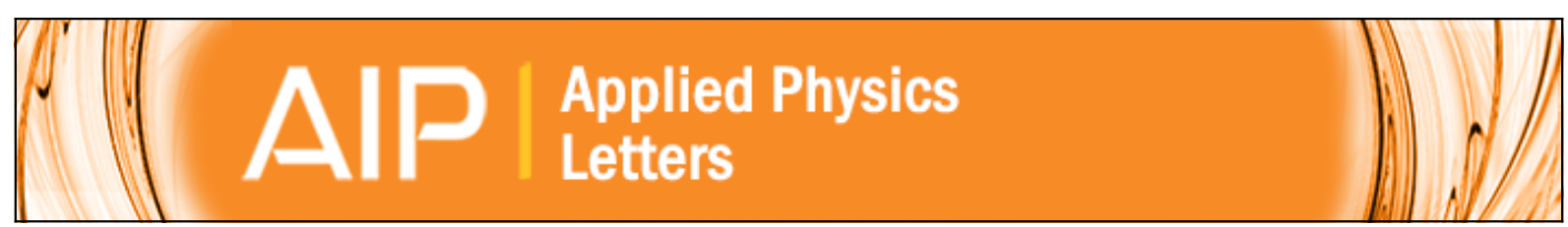

\title{
Bending kinetics of a photo-actuating nematic elastomer cantilever
}

N. Torras, K. E. Zinoviev, J. E. Marshall, E. M. Terentjev, and J. Esteve

Citation: Applied Physics Letters 99, 254102 (2011); doi: 10.1063/1.3670502

View online: http://dx.doi.org/10.1063/1.3670502

View Table of Contents: http://scitation.aip.org/content/aip/journal/apl/99/25?ver=pdfcov

Published by the AIP Publishing

$\underset{\substack{\text { Aubloning } \\ \text { Alp }}}{A}$ Re-register for Table of Content Alerts

Create a profile.

Sign up today! 


\title{
Bending kinetics of a photo-actuating nematic elastomer cantilever
}

\author{
N. Torras, ${ }^{1}{ }^{1, a)}$ K. E. Zinoviev, ${ }^{1}$ J. E. Marshall, ${ }^{2}$ E. M. Terentjev, ${ }^{2}$ and J. Esteve ${ }^{1}$ \\ ${ }^{1}$ IMB-CNM (CSIC), Campus UAB, 08193 Bellaterra, Barcelona, Spain \\ ${ }^{2}$ Cavendish Laboratory, University of Cambridge, J. J. Thomson Avenue, Cambridge CB3 OHE, UK
}

(Received 24 October 2011; accepted 25 November 2011; published online 19 December 2011)

\begin{abstract}
Liquid crystal elastomers (LCE) containing embedded carbon nanotubes (CNTs) contract when exposed to light, due to LC disordering induced by the ability of CNTs to absorb light and convert it into thermal energy. A cantilever made of LCE-CNTs exposed to light demonstrates dynamic bending due to inhomogeneous strain distribution caused by exponential heat generation across the cantilever width. Analysis of bending dynamics helps to extract parameters that are important for designing actuators based on these materials. In this work, we have carried out direct measurements of temperature evolution inside the cantilever and related its kinetics to the applied irradiation power. (C) 2011 American Institute of Physics. [doi:10.1063/1.3670502]
\end{abstract}

Liquid crystal elastomers (LCEs) are a unique class of materials, which have been generating considerable research interest in recent years due to their remarkable ability to reversibly change their bulk dimensions in response to heat or light. ${ }^{1}$ This ability makes LCEs attractive candidates for a variety of applications in robotics, photonics, and microfluidics. It has been demonstrated that modified LCEs can exhibit thermal, ${ }^{2-5}$ electrical, ${ }^{6}$ and photo-induced ${ }^{7,8}$ actuation; based on these achievements, a number of devices such as microvalves, ${ }^{9}$ optical tweezers, and microgrippers ${ }^{10}$ among others have been reported. Photo-induced actuation in particular is attractive because it can be triggered by an external "remote" stimulus (rather than by direct heating, which is inconvenient for most practical applications) and because the speed and periodicity of the stimulus can be finely controlled. In order to achieve photo-actuation in LCEs, lightabsorbing components, such as carbon nanotubes (CNTs), azobenzene moieties, or dyes sensitive to ultraviolet (UV) and visible light must be incorporated into the polymer matrix. One useful property of CNTs is their ability to absorb light and convert it into heat energy; ${ }^{11}$ by incorporating them into the LCE polymer matrix, we are essentially using the CNTs as an alternative method of providing heat to the LCE. CNTs also have a high thermal conductivity along their principal axis, which can also help with heat transport through the material, decreasing the response time, and potentially improving the material properties of the composite. However, the relationship between added CNTs and the thermal properties of the material is complex, since the characteristics of the composite depend not only on added CNT concentration but also on the method by which CNTs are dispersed through the polymer. ${ }^{12} \mathrm{CNTs}$ allow actuation of the composite to be triggered by light at any wavelength in the visible and infrared range, ${ }^{13}$ since the mechanism of actuation is thermal and relies only on the amount of the absorbed electromagnetic energy.

The chemical synthesis and processing of LCEs and LCE composites has been extensively reported elsewhere. Characterisation techniques have been demonstrated that can

\footnotetext{
a)Electronic mail: nuria.torras@imb-cnm.csic.es.
}

measure the induced strain (or induced stress at constant strain) of the LCE-CNT composite material when it is heated or illuminated with a light source. ${ }^{11}$

In this article, we report the results of experiments focused on the bending dynamics of a LCE-CNTs cantilever illuminated from one side. The bending kinetics of an LCE cantilever exposed to heat has been discussed elsewhere, including the physics behind the cantilever motion. ${ }^{15,16}$ Hon and coworkers used a cantilever made from a "neat" LCE material (containing no added CNTs) and focused on the response of that cantilever to direct heat. We will herein concentrate on cantilevers made from an LCE-CNT composite and will discuss factors such as heat propagation through the cantilever, time constants, and uniformity of the material in the case where heat is not being provided by a direct heat source but by the light-irradiated CNTs embedded in the polymer.

A cantilever is a versatile mechanical structure, which can be used to obtain information about the material from which it is made. We used thin strips of mono-domain nematic side-chain LCEs, containing a low concentration of added CNTs (0.1 wt. \%), with thicknesses of $0.3-0.6 \mathrm{~mm}$. The LC mesogens in the LCE were aligned using the twostep cross-linking process based on the mechanical stretching method pioneered by Küpfer and Finkelmann in $1991{ }^{3}$ and CNTs were incorporated into the composite material using the method outlined by Ji et al. ${ }^{14}$

First, measurements of the variation in natural length $L$ of our LCE-CNT material with temperature were made using images of a $1.2 \times 1.2 \mathrm{~mm}^{2}$ sample obtained with an optical microscope. The sample was positioned horizontally without an applied load and heated at average rate of $4 \mathrm{~K} / \mathrm{min}$ in a miniaturized oven. The measured variation in $L(T)$ was fitted (see Fig. 1) to a function given by the expression

$$
L(T) / L\left(T_{s}\right)=1+0.29\left(1-T / T_{s}\right)^{0.15},
$$

where $L\left(T_{s}\right)$ is the length of the sample in the isotropic state, i.e., when it is fully contracted. $T_{s}=350 \mathrm{~K}$ is the nematicisotropic transition temperature. The exponential coefficient in Eq. (1) does not match closely to results quoted in literature, where its value ranges between 0.19 and 0.29 ; but it fits well to the experimental data measured in nematic state. 


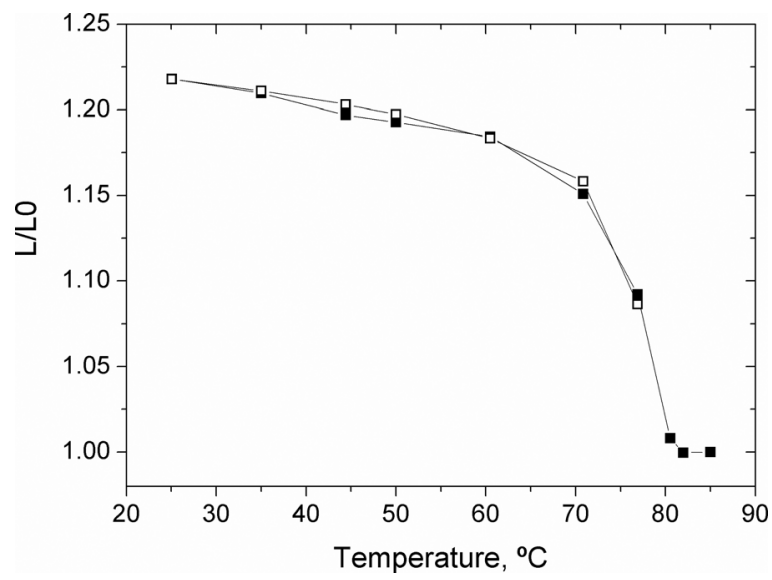

FIG. 1. Temperature dependence of the material contraction and fitting according to Eq. (1).

Based on these data, we proceed with the characterisation of the cantilever cut out of an LCE strip with thickness of $0.5 \mathrm{~mm}$. The sample was cut into a rectangular strip $4 \mathrm{~mm} \times$ $1.2 \mathrm{~mm}$ at room temperature and fixed from one end to form a cantilever beam. The cantilever was placed in a plastic box to avoid cooling due to air convection. A laser diode (LD) coupled to a current controller provided irradiation (wavelength $660 \mathrm{~nm}$ ) to the strip, allowing rapid ON/OFF switching. The light emitted by the LD had a Gaussian intensity distribution with wavelength; also a divergence of $30^{\circ}$ and $10^{\circ}$, in the vertical ( $\mathrm{Y}$ axis) and horizontal ( $\mathrm{X}$ axis) directions, respectively, which correspond to $0.9 \mathrm{~mm}$ and $2.7 \mathrm{~mm}$ full width at half maximum (FWHM) of the spot on the plane of the cantilever front surface. The surface exposed to the irradiation was located $5 \mathrm{~mm}$ from the LD and aligned symmetrically with respect to the center of the spot (see Fig. 2(a)). Linear absorption of photons by the CNTs incorporated in rubber results in exponential attenuation of light with distance. The energy of photons is converted thus into thermal energy. Light absorption characteristic length was estimated in another experiment, using collimated light of known intensity directed toward the sample and measuring
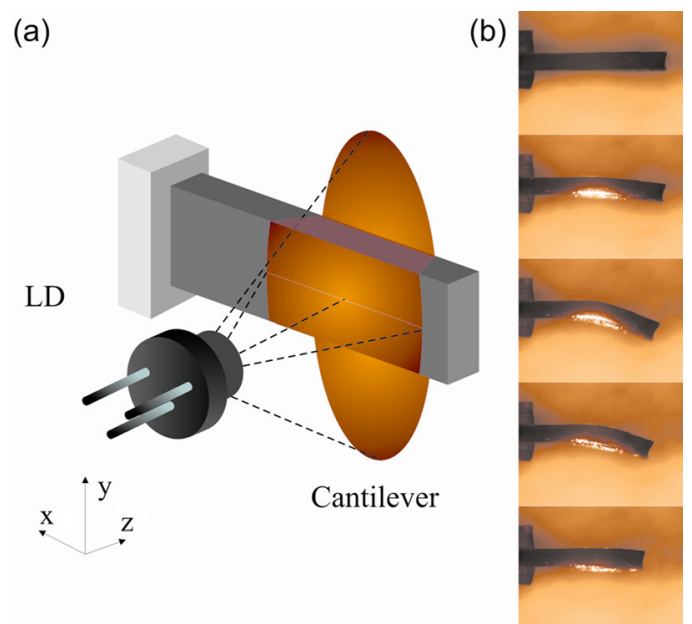

FIG. 2. (Color online) (a) Schematics of the experiment; (b) top view images of the cantilever (Y axis). The images were obtained at $0,2,4,8$, and $14 \mathrm{~s}$ after the LD was switched on, with $D_{p}$ fixed at $2.25 \mathrm{~W} \mathrm{~cm}^{-2}$. The cantilever on the photos was $4 \mathrm{~mm}$ long and $0.5 \mathrm{~mm}$ thick. After irradiation, it is $3.2 \mathrm{~mm}$ long-contraction 1.25 - and $0.6 \mathrm{~mm}$ thick-expansion 1.2 - the intensity of light transmitted through the film. We assumed that the material was uniform and that light exponentially decayed inside the sample, in accordance with the Beer-Lambert law. The absorption length $\alpha$ of $60 \mu \mathrm{m}$ was obtained by calculation. This gives rise to the result that more than $90 \%$ of the incident light energy is absorbed inside a $150 \mu \mathrm{m}$ thick layer.

Movies of the cantilever bending motion were recorded by a conventional optical microscope camera and then manually analysed. The temperature distribution over the cantilever top face was measured twice per second by a FLIR SC 5000-series infrared (IR) camera provided with ALTAIR software, with $30 \mu \mathrm{m}$ of resolution per pixel. The emissivity of the sample was assumed to be 0.7 . This value was adjusted to fit the temperature of contraction of the material obtained during the experiment with thermal actuation (see expression (1)) to the temperature of contraction measured by the IR camera. When exposed to the irradiation at a constant power the cantilever starts bending and after some time comes to a steady state, Fig. 2(b), which is a function of the applied power.

We can distinguish between three modes of bending, characterized by the cantilever curvature $(C)$ in steady state, as function of power density $\left(D_{P}\right)$ in the center of the spot: (i) $C$ increases with power, while $D_{P}<1.16 \mathrm{~W} \mathrm{~cm}^{-2}$; (ii) $C$ decreases with power when $1.16 \mathrm{~W} \mathrm{~cm}^{-2}<D_{P}<2.1 \mathrm{~W}$ $\mathrm{cm}^{-2}$; (iii) the cantilever becomes flat in steady state when $D_{P}>2.1 \mathrm{~W} \mathrm{~cm}^{-2}$.

During the experiment, as $D_{p}$ increases, there comes a point where an interface exists between an area of the surface where temperatures are above $T_{s}$ and an area where the temperatures are below $T_{s}$; at this stage, the cantilever thickness experiences a quick change. The thickness increases by nearly $20 \%$ in the segment with the higher temperature. A typical temperature distribution over a cantilever illuminated with a power density of $2.25 \mathrm{~W} \mathrm{~cm}^{-2}$ is shown in Fig. 3(a). The temperature distribution is highly asymmetrical across the cantilever in the plane of the spot center, at $x=x_{0}$, becoming more uniform away from $x_{0}$. Experimentally measured temperature evolution in two points located on the front and reverse sides of the cantilever at $x=x_{0}$ are demonstrated in Fig. 3(b). In the steady state, starting from second 12 , the difference in temperature between both sides is about $10^{\circ}$. There is a significant delay of about $1 \mathrm{~s}$ before the temperature on the reverse side starts to increase at the same rate as the temperature in front.

The temperature evolution inside the cantilever is governed by the heat equation, whose dimensionality can be reduced if we neglect inhomogeneous light distribution in one direction. Light incident on the cantilever is considered uniform along the $\mathrm{Y}$ axis, while having a Gaussian distribution with mean value $x_{0}$ and variance $\mu$ in $\mathrm{X}$ direction. As the divergence of light exiting the LD is high (nearly $30^{\circ}$ ) in the vertical direction, we can assume (to a first approximation) that the cantilever is homogeneously illuminated from top to bottom, resulting in the relation

$$
C_{v} \frac{\partial T}{\partial t}-k_{f}\left(\frac{\partial^{2} T}{\partial z^{2}}+\frac{\partial^{2} T}{\partial x^{2}}\right)=\theta_{0} \exp \left(-\frac{z}{\alpha}\right) \exp \left(-\left(\frac{x-x_{0}}{\mu}\right)^{2}\right)
$$


(a)

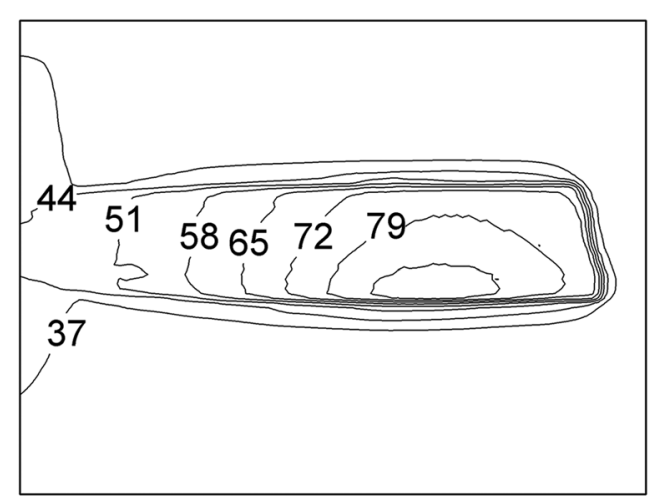

(b)

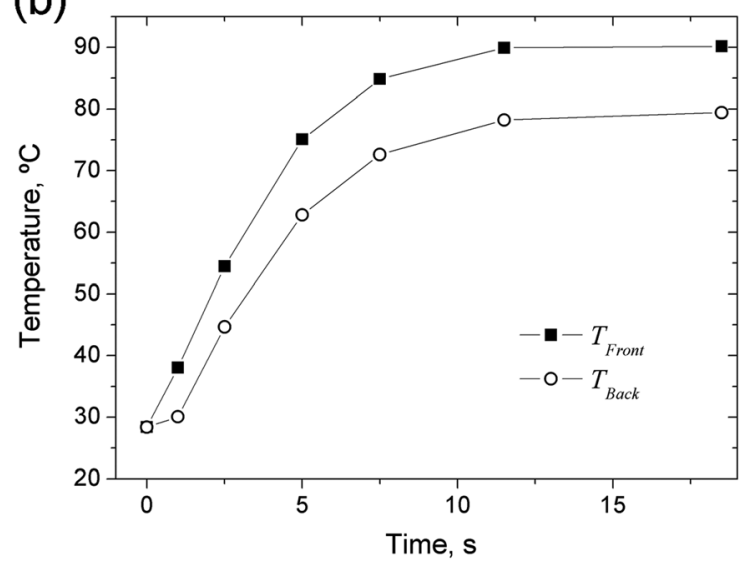

FIG. 3. (a) Two dimensional stationary temperature distribution over the cantilever measured by IR camera; (b) Temperature evolution measured by IR camera. Squares represent temperature on the front (illuminated) side; circles depict temperature evolution on the back side of the cantilever. The LD power was fixed at $2.25 \mathrm{Wcm}^{-2}$.

where $\theta_{0}=P_{0} / \alpha\left(\mathrm{W} \mathrm{m}^{-3}\right)$ stands for heat density on the front side.

It is difficult to solve this equation analytically, so numerical analysis was performed using commercially available software. We assume $P_{0}\left(\mathrm{~W} \mathrm{~m}^{-2}\right)$ to be the power flux density of light incident on the cantilever in the center of the spot.

To simulate the temperature distribution around the cantilever, three different cases can be distinguished, which have been already mentioned above (Fig. 4):

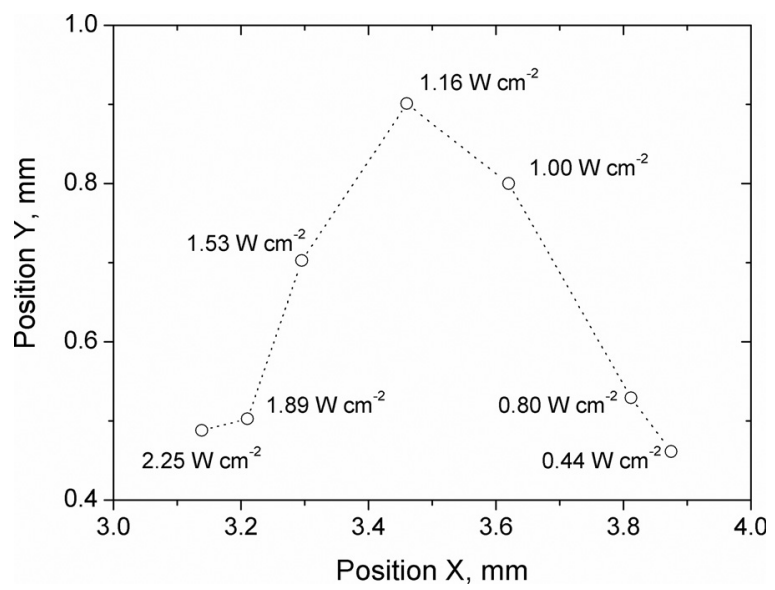

FIG. 4. Cantilever upper apex position coordinates in steady state at different irradiation powers.
Mode (a): The cantilever curvature increases with the irradiating power. At constant power, immediately after the experiment starts, the cantilever curvature increases with time. This mode disappears when the isotherm of $T_{s}$ crosses both the front and the back surfaces.

Mode (b): In this mode, when the cantilever is illuminated at constant power, there is an initial increase of curvature with time. After that the curvature decreases until it reaches a steady state value. When the isotherm $T s$ crosses the sample from the front to the back a portion of the cantilever has zero stress gradient and zero curvature. Further power increase just amplifies this segment of fully contracted material.

Mode (c): The cantilever curvature decreases with time and then drops down to zero. No stress gradient can be found in the cross sections where the temperature is more than $T_{s}$. The isotherms of temperatures below $T s$ become symmetrical in cross section, and no bending is produced.

It is worth noting that the relaxation of the cantilever does not follow the same kinetics. The elongation of a fully contracted cantilever is not accompanied by bending at all, because when the light is switched off the temperature gradient inside the cantilever quickly disappears. The data on bending kinetics of a rectangular beam like the one demonstrated in this work can be used for characterization of elastomers. Accurate simulations of heat propagation are required to extract important physical properties of the material using this testing technique, which is the subject of our future work.

This work has been supported by EU FP7 funding program under contract NMP 228916. We thank Miquel Vellvehí for his help in the experiments with the IR camera.

${ }^{1}$ M. Warner and E. M. Terentjev, Liquid Crystal Elastomers (Oxford University Press, New York, 2003).

${ }^{2}$ J. Küpfer and H. Finkelmann, Makromol. Chem., Rapid Commun. 12, 717 (1991).

${ }^{3}$ A. R. Tajbakhsh and E. M. Terentjev, Eur. Phys. J. E 6, 181 (2001).

${ }^{4}$ D. L. Thomsen, P. Keller, J. Naciri, R. Pink, H. Jeon, D. Shenoy, and B. R. Ratna, Macromolecules 34, 5868 (2001).

${ }^{5}$ K. A. Burke and P. T. Mather, J. Mater. Chem. 20, 3449 (2010).

${ }^{6}$ M. Chambers, H. Finkelmann, M. Remkar, A. Sanchez-Ferrer, B. Zalar, and S. Zumer, J. Mater. Chem. 19, 1524 (2008).

${ }^{7}$ H. Finkelmann, E. Nishikawa, G. G. Pereira, and M. Warner, Phys. Rev. Lett. 87, 015501 (2001).

${ }^{8}$ P. M. Hogan, A. R. Tajbakhsh, and E. M. Terentjev, Phys. Rev. E 65, 041720 (2002).

${ }^{9}$ F. Benito-Lopez, R. Byrne, A. M. Raduta, N. E. Vrana, G. McGuinness, and D. Diamond, Lab Chip 10, 195 (2010).

${ }^{10}$ A. Sánchez-Ferrer, T. Fischl, M. Stubenrauch, H. Wurmus, M. Hoffmann, and H. Finkelmann, Macromol. Chem. Phys. 210, 1671 (2009).

${ }^{11}$ P. M. Ajayan, M. Tettones, A. de la Guardia, V. Huc, N. Grobert, B. Q. Wei, H. Lezec, G. Ramanath, and T. W. Ebbesen, Science 296, 705 (2002).

${ }^{12}$ Yan Y. Huang and E. M. Terentjev, Adv. Funct. Mater. 20, 4062 (2010).

${ }^{13}$ N. Torras, C. J. Camargo, K. Zinoviev, H. Campanella, J. E. Marshall, E. M. Campo, E. M. Terentjev, and J. Esteve, in Proceedings of SPIE Conference on Nano-Opto-Mechanical Systems, SPIE, San Diego, CA, August 21-25 (2011), Vol. 8107, 810704.

${ }^{14}$ Yan Ji, Yan Y. Huang, Rakchnanok Rungsawang, and E. M. Terentjev, Adv. Mater. 22, 3436 (2010).

${ }^{15}$ K. K. Hon, D. Corbett, and E. M. Terentjev, Eur. Phys. J. E 25, 83 (2008).

${ }^{16}$ M. Warner and L. Mahadevan, Phys. Rev. Lett. 92, 134302 (2004). 